(W)

Check for

updatos

Cite as

Nano-Micro Lett.

(2022) 14:26

Received: 30 September 2021

Accepted: 2 November 2021

Published online: 10 December 2021

(C) The Author(s) 2021

\section{Hierarchically Multifunctional Polyimide Composite Films with Strongly Enhanced Thermal Conductivity}

\author{
Yongqiang Guo ${ }^{1}$, Hua Qiu ${ }^{1}{ }^{凶}$, Kunpeng Ruan ${ }^{1}$, Yali Zhang ${ }^{1}$, Junwei Gu ${ }^{1 凶}$
}

\title{
HIGHLIGHTS
}

- Hierarchically multifunctional polyimide composite films were fabricated by hierarchical design and assembly strategy.

- Polyimide composite films have three functional layers and integrates high thermal conductivity $\left(95.40 \mathrm{~W}(\mathrm{~m} \mathrm{~K})^{-1}\right)$, excellent EMI shielding (34.0 dB) and good tensile strength (93.6 MPa).

- Polyimide composite films present broad application prospects in electronics fields according to the test results in the central processing unit.

ABSTRACT The development of lightweight and integration for electronics requires flexible films with high thermal conductivity and electromagnetic interference (EMI) shielding to overcome heat accumulation and electromagnetic radiation pollution. Herein, the hierarchical design and assembly strategy was adopted to fabricate

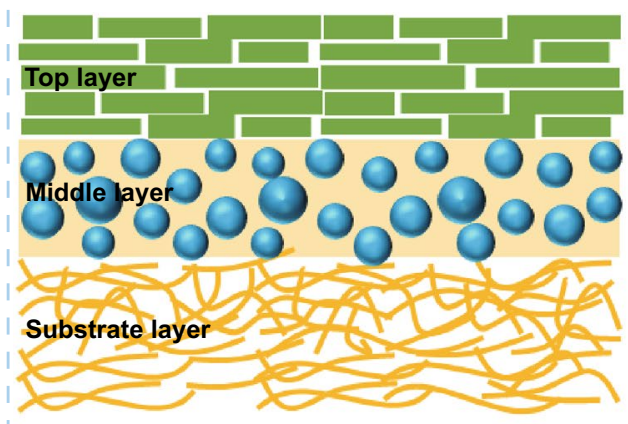

$\mathrm{GO} / \mathrm{EG} \bigcirc \mathrm{Fe}_{3} \mathrm{O}_{4} \quad \mathrm{PI}$ matrix $\mathrm{X} \mathrm{PI}$ fibers

Polyimide composite films

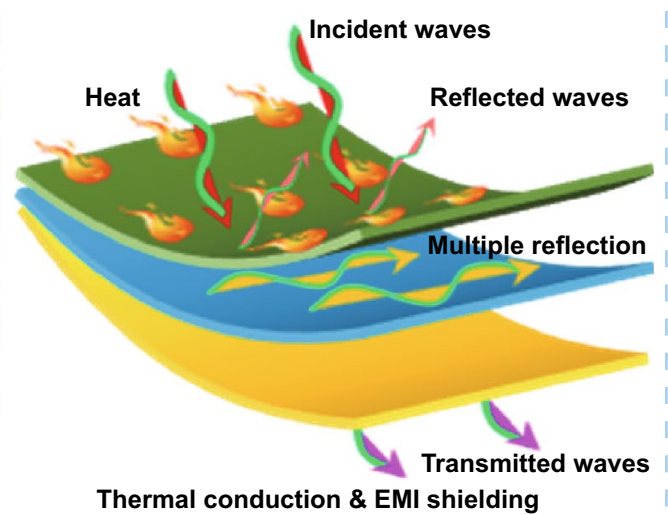

Thermal conduction \& EMI shielding hierarchically multifunctional polyimide composite films, with graphene oxide/expanded graphite (GO/EG) as the top thermally conductive and EMI shielding layer, $\mathrm{Fe}_{3} \mathrm{O}_{4} /$ polyimide $\left(\mathrm{Fe}_{3} \mathrm{O}_{4} / \mathrm{PI}\right)$ as the middle EMI shielding enhancement layer and electrospun PI fibers as the substrate layer for mechanical improvement. PI composite films with $61.0 \mathrm{wt} \%$ of GO/EG and $23.8 \mathrm{wt} \%$ of $\mathrm{Fe}_{3} \mathrm{O}_{4} / \mathrm{PI}$ exhibits high in-plane thermal conductivity coefficient $\left(95.40 \mathrm{~W}\left(\mathrm{~m} \mathrm{~K}^{-1}\right)\right.$, excellent EMI shielding effectiveness (34.0 dB), good tensile strength $(93.6 \mathrm{MPa})$ and fast electric-heating response $(5 \mathrm{~s})$. The test in the central processing unit verifies PI composite films present broad application prospects in electronics fields.

KEYWORDS EMI shielding; Flexible films; Polyimide; Thermal conductivity

Hua Qiu, huaqiu@nwpu.edu.cn; Junwei Gu, gjw@nwpu.edu.cn; nwpugjw@163.com

1 Shaanxi Key Laboratory of Macromolecular Science and Technology, School of Chemistry and Chemical Engineering, Northwestern Polytechnical University, Xi' an 710072, Shaanxi, People's Republic of China 


\section{Introduction}

The development of lightness and high integration for electronics inevitably causes serious heat accumulation $\left(>5 \mathrm{~W} \mathrm{~cm}^{-2}\right)[1]$ and electromagnetic interference (EMI), which trigger increasing demand for effective heat dissipation and EMI shielding at the multifunctional materials level [2-4]. In this context, polymer-based composite films integrating excellent thermal conduction and EMI shielding are expected to meet the requirement of electronics manufacturing due to their advantages of lightweight, easy processing and good designability [5-7].

Many single or hybrid fillers with different functions (thermal, electrical, magnetic [8-10]) have been added into polymer matrix by molding press, filtration, blade coating, etc. [11-13], to fabricate polymer-based composite films with enhanced thermal conduction and EMI shielding performances [14-18]. Wei et al. prepared multi-walled carbon nanotube/water-based polyurethane (MWCNT/WPU) composite films by in situ polymerization and blade coating method. In the presence of $48.1 \mathrm{wt} \%$ MWCNT, the in-plane thermal conductivity coefficient ( $\lambda$ II) and EMI shielding effectiveness (SE) of MWCNT/ WPU composite films with thickness of $80 \mu \mathrm{m}$ reached $5.31 \mathrm{~W}$ $(\mathrm{m} \mathrm{K})^{-1}$ and $65.3 \mathrm{~dB}$, respectively [19]. Fu et al. filtrated the mixture of graphene oxide (GO) and cellulose nanofibers (CNF) and reduced it to prepare rGO/CNF composites films. The $\lambda \|$ and EMI SE of the $\mathrm{rGO} / \mathrm{CNF}$ composite films with $50 \mathrm{wt} \% \mathrm{rGO}$ were $7.30 \mathrm{~W}(\mathrm{~m} \mathrm{~K})^{-1}$ and $26.2 \mathrm{~dB}$, respectively [20]. However, directly mixing the fillers and polymer has obvious disadvantages, which is difficult to form efficient fillers thermal conduction pathways and networks because the fillers are easily separated or coated by the polymer matrix [21]. Moreover, high interfacial thermal resistance between the fillers and polymer matrix results in low thermal conductivity for the prepared composites. In addition, introduction of a large number of thermally conductive fillers is likely to cause poor mechanical and processing properties and higher cost and density, and the enhancement in thermal conductivity is relatively limited [22].

To solve the abovementioned problems, researchers directly construct fillers thermal conduction pathways to prepare fillers films, and then combining them with polymer to fabricate hierarchical composite films [23]. Wang et al. attached graphite nanosheets (GNPs) to nylon gauze by filtration method, and then prepared two-layer structured
GNPs/nylon composite films by combing hot pressing. When the amount of GNPs was $11.8 \mathrm{wt} \%$, the $\lambda \|$ and EMI SE of the GNPs/nylon composite film reached $15.8 \mathrm{~W}(\mathrm{~m} \mathrm{~K})^{-1}$ and $58.1 \mathrm{~dB}$, which were 63 times and 157 times that of pure nylon, respectively. However, its tensile strength was only 32.0 Mpa [24]. Liu et al. prepared multi-hierarchical structured GNPs/CNF composite films by alternate filtration method. The $\lambda \|$, EMI SE and tensile strength of the GNPs/ CNF composite film with $25 \mathrm{wt} \%$ GNPs were $33.55 \mathrm{~W}$ $(\mathrm{m} \mathrm{K})^{-1}, 27.4 \mathrm{~dB}$ and 122.4 MPa, respectively [25]. However, these hierarchical composite films are easy to peel off and have poor durability due to the weak force among the fillers [26].

Electrospun polymer fibers have diameters ranging from sub-nanometers to micrometers, the high orientation of polymer molecular chains gives the fibers good mechanical properties and excellent flexibility [27]. Hou et al. prepared electrospun polyimide (PI) fibers to reinforce epoxy resin (EP). The tensile strength of PI/EP composites with $30 \mathrm{wt} . \%$ PI fibers was as high as $227.3 \mathrm{MPa}$, which was $511 \%$ higher than that of pure EP (37.2 MPa). Therefore, the introduction of polymer fibers into composite films is expected to improve their mechanical properties further [27].

Expanded graphite (EG) possesses excellent thermal conductivity and outstanding EMI shielding performances. However, its brittle nature, difficult assembly and poor mechanical properties limit the preparation and wide application of EG film [28, 29]. To our knowledge, GO has high specific area and could form $\pi$ - $\pi$ interaction with EG [30]. The stable dispersion ability of GO in water can promote the dispersion of EG and reduce its agglomeration [31]. Flexible GO films have been proven to be dense and can sustain repeated bending, which could endow EG film with good mechanical properties and flexibility via forming hetero-sheet bonding and introducing foldable and stretchable wrinkles. Besides, excellent thermal conductivity and outstanding EMI shielding of EG film could also be maintained. However, high electrical conductivity of EG can cause excessive reflectivity in electromagnetic waves, resulting in secondary radiation to the environment $[8,32]$. It's necessary to increase the loss of electromagnetic waves to reduce secondary radiation pollution [33], such as combining magnetic fillers with EG [34].

Herein, a hierarchical design and assembly strategy was adopted to fabricate hierarchically multifunctional PI 
composite films with high thermal conductivity, excellent EMI shielding and good mechanical properties. The top layer of PI composite films was GO/EG, fabricated by the filtration and molding press method, whose main function was thermal conduction and EMI shielding. The introducing of GO into EG films solved the brittle nature of EG films, leading to the balance of mechanical properties, thermal conductivity and EMI shielding performances by optimizing the ratio of GO and EG. The substrate layer of PI composite films was electrospun PI fibers to enhance the mechanical properties. The top and substrate layer were combined with $\mathrm{Fe}_{3} \mathrm{O}_{4} / \mathrm{PI}$ prepared by in situ polymerization and blade coating, improving the EMI shielding performance. The effects of the amount of each layer on the thermal conductivity, EMI $\mathrm{SE}$, mechanical properties and the electric-heating response of the PI composite films were analyzed. In addition, the potential application of the films in thermal management was also demonstrated by experimental test.

\section{Experimental Methods}

\subsection{Materials}

Graphite (> 100 mesh, 99.95\%) and ammonium persulphate $\left(\left(\mathrm{NH}_{4}\right)_{2} \mathrm{~S}_{2} \mathrm{O}_{8}, 99 \%\right)$ were both purchased from Shanghai Aladdin Bio-Chem Technology Co., Ltd., China. Ferroferric oxide $\left(\mathrm{Fe}_{3} \mathrm{O}_{4}, 99.5 \%\right)$ was provided by Shanghai Macklin Biochemical Co., Ltd., China. Pyromellitic dianhydride (PMDA, 99\%) and 4, 4'-oxydianiline (ODA, 99\%) were both supplied by Beijing J\&K Scientific Co., Ltd., China. Dimethylacetamide (DMAc, 99\%) was purchased from Guangdong Guanghua Technique Co., Ltd., China. Concentrated sulfuric acid $\left(\mathrm{H}_{2} \mathrm{SO}_{4}, 95-98 \%\right)$, potassium permanganate $\left(\mathrm{KMnO}_{4}\right)$ and hydrogen peroxide $\left(\mathrm{H}_{2} \mathrm{O}_{2}, 30 \%\right)$ were all supplied by Beijing Chemical Works, China. All materials were used without further purification.

\subsection{Preparation of GO/EG Films}

GO was prepared by the Hummers method [28]. Graphite was treated by the $\left(\mathrm{NH}_{4}\right)_{2} \mathrm{~S}_{2} \mathrm{O}_{8}$ to prepare EG [35]. $2 \mathrm{~g}$ of graphite and $80 \mathrm{~mL}$ of $\mathrm{H}_{2} \mathrm{SO}_{4}$ were mixed and stirred at $35{ }^{\circ} \mathrm{C}$, and then $10 \mathrm{~g}$ of $\left(\mathrm{NH}_{4}\right)_{2} \mathrm{~S}_{2} \mathrm{O}_{8}$ was added into the mixture and stirred for $1 \mathrm{~h}$. The mixture was diluted with $100 \mathrm{~mL}$ deionized water followed with filtration, washing and desiccation to prepare EG. Then, EG was re-dispersed in $\mathrm{GO}$ aqueous solution ( 1 or $2 \mathrm{mg} \mathrm{mL}^{-1}$ ) and ultrasonicated for $30 \mathrm{~min}$, the amount of $E G$ was 3, 5, 7, 9 or $11 \mathrm{mg} \mathrm{mL}^{-1}$. A certain amount of suspension was further filtrated, dried and molding press (Ambient temperature, $20 \mathrm{MPa}$ ) to fabricate compacted GO/EG films (diameter of $40 \mathrm{~mm}$ ).

\subsection{Preparation of Electrospun PI Fibers}

Equimolar ODA and PMDA were added into DMAc successively, and stirred for $30 \mathrm{~min}$ at ice bath to get PAA solution. PAA fibers were obtained with the electrospinning technology as follows: collection distance of $30 \mathrm{~cm}$, voltage of $25 \mathrm{kV}$, injection speed of $0.05 \mathrm{~mm} \mathrm{~min}^{-1}$. PAA fibers were dried for $12 \mathrm{~h}$ at $80^{\circ} \mathrm{C}$ and then thermal imidized $\left(120^{\circ} \mathrm{C}\right.$ $/ 1 \mathrm{~h}+200{ }^{\circ} \mathrm{C} / 1 \mathrm{~h}+250{ }^{\circ} \mathrm{C} / 1 \mathrm{~h}$, heating rate of $1^{\circ} \mathrm{C} \mathrm{min}{ }^{-1}$ ) to get PI fibers.

\subsection{Preparation of PI Composite Films}

A certain amount of ODA and $\mathrm{Fe}_{3} \mathrm{O}_{4}$ was dispersed in DMAc; the PMDA with equal molar of ODA was added to the above solution and stirred for $30 \mathrm{~min}$ at ice bath to get $\mathrm{Fe}_{3} \mathrm{O}_{4} / \mathrm{PAA}$ mixture (the mass fraction of $\mathrm{Fe}_{3} \mathrm{O}_{4}$ in $\mathrm{Fe}_{3} \mathrm{O}_{4} /$ PAA was $0 \%, 10 \%, 20 \%, 30 \%$ or $40 \%$ ). $\mathrm{Fe}_{3} \mathrm{O}_{4} / \mathrm{PAA}$ was poured on the GO/EG films and spread out by the blade coating, and then put $\mathrm{PI}$ fibers on the $\mathrm{Fe}_{3} \mathrm{O}_{4} / \mathrm{PAA}$. The whole composites were dried for $12 \mathrm{~h}$ at $80^{\circ} \mathrm{C}$ and then imidized with the same procedure of PI fibers to get PI composite films. The components of PI composite films are shown in Table S1.

\subsection{Characterization}

Morphologies of PI composite films were observed by scanning electron microscope (SEM, Verios G4, FEI Corporation, USA). The $\lambda$ of PI composite films was measured by the transient plane heat source method (TPS2200, Hot Disk, Sweden) according to ISO 22007-2. Slab and thin film module were chosen to calculate the in-plane and throughplane $\lambda$ of PI composite films. The surface temperature of PI composite films was recorded by infrared thermography (Ti 300, Fluke, USA). Conductivity tester (RTS-8, Guangzhou Four Probes Technology, China) and Physical Property 
Measurement System (CFMS-14T, Cryogenic, UK) were utilized to measure the electrical conductivity and magnetic properties. EMI shielding parameters of PI composite films were tested by VNA (MS4644A, Anritsu, Japan) at X-band (8.2-12.4 GHz) according to ASTM D5568-08. The mechanical properties of PI composite films were tested by electronic universal testing machine (CMT 7204, Suns, China) in accordance with ASTM D882-2018. The tensile rate was $2 \mathrm{~mm} \mathrm{~min}^{-1}$, and each sample was measured at least 5 times; the average value was taken.

\section{Results and Discussions}

Schematic diagram of the preparation process for PI composite films is shown in Fig. 1. The top layer of PI composite films is GO/EG. EG sheet is thicker than GO (Fig. S1), maintaining high $\mathrm{C} / \mathrm{O}$ atomic ratio (Fig. S3a) and complete crystal structure (Fig. S3b), giving the GO/EG films excellent thermal conductivity and EMI shielding performances [36]. GO sheet seems like flexible cloth (Fig. S1b), which gives the GO/EG film formability and flexibility [37]. As GO has poor thermal conductivity [24], the amount of GO in
GO/EG should be as low as possible to make GO/EG obtain high thermal conductivity. In this work, a certain amount of EG was added into the 1 or $2 \mathrm{mg} \mathrm{mL}^{-1} \mathrm{GO}$ aqueous solutions to judge the formability of GO/EG films. Results show that even a little EG (three times the mass of GO) is added into the $1 \mathrm{mg} \mathrm{mL}^{-1} \mathrm{GO}$ solution, the GO/EG films are difficult to completely strip off from the filter membrane (Fig. S1c). When the EG whose mass is 5.5 times that of $\mathrm{GO}$ is added into the $2 \mathrm{mg} \mathrm{mL}^{-1} \mathrm{GO}$ solution, the GO/EG films are brittle (Fig. S1e). Therefore, the optimized mass ratio of $\mathrm{GO}$ and $\mathrm{EG}$ is $2: 9$ and the $\mathrm{GO}$ aqueous solution is $2 \mathrm{mg} \mathrm{mL}^{-1}$, the fabricated GO/EG films could be completely peeled off from the filter membrane (Fig. S1d). In addition, the formability of GO/EG films is also related to the volume of $\mathrm{GO} / \mathrm{EG}$ dispersion during filtration. In this work, the filter membrane cannot be covered if the volume of GO/EG dispersion is less than $3 \mathrm{~mL}$. However, when the volume of $\mathrm{GO} / \mathrm{EG}$ dispersion exceeds $9 \mathrm{~mL}$, the $\mathrm{GO} / \mathrm{EG}$ films are too thick to be completely peeled off. The thickness of GO/ EG films prepared by 3, 5, 7 and $9 \mathrm{~mL}$ of GO/EG dispersion is approximately 20, 35, 50, and $70 \mu \mathrm{m}$ (Fig. S4a-d, Table S1). The thickness of the PI fibers mat is about $160 \mu \mathrm{m}$

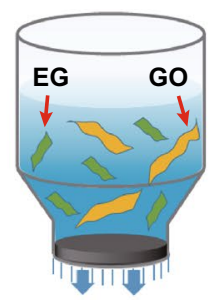

GO+EG dispersion

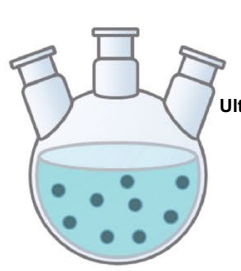

$\mathrm{Fe}_{3} \mathrm{O}_{4}+\mathrm{ODA}$

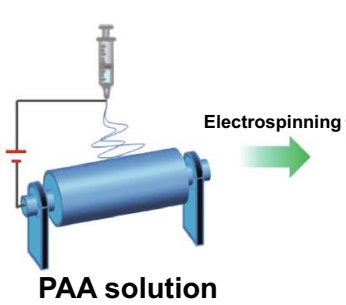

\section{然}
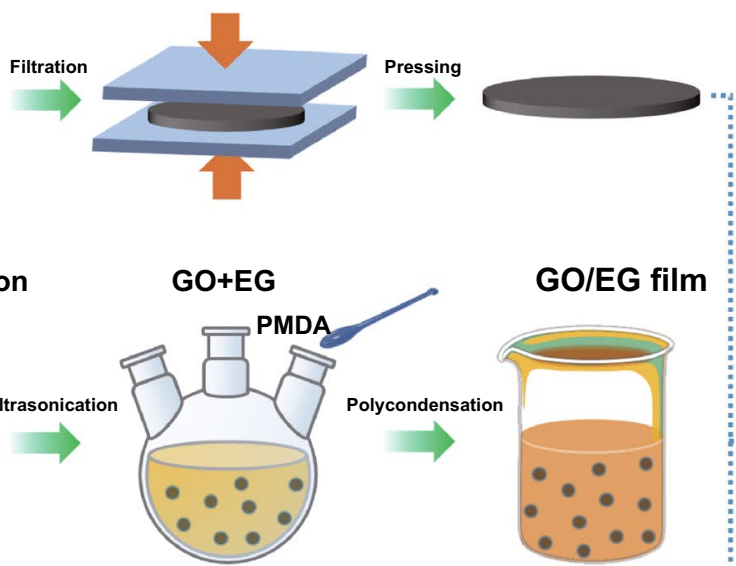

$\mathrm{Fe}_{3} \mathrm{O}_{4}+\mathrm{ODA}+\mathrm{PMDA}$

GO/EG film

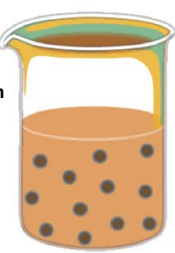

$\mathrm{Fe}_{3} \mathrm{O}_{4} / \mathrm{PAA}$
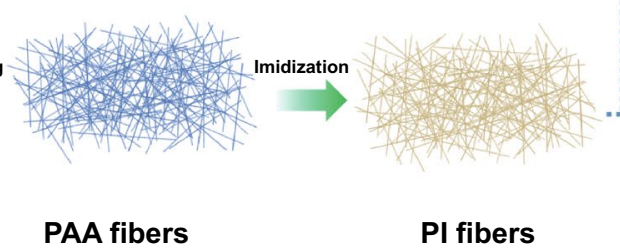

PI fibers

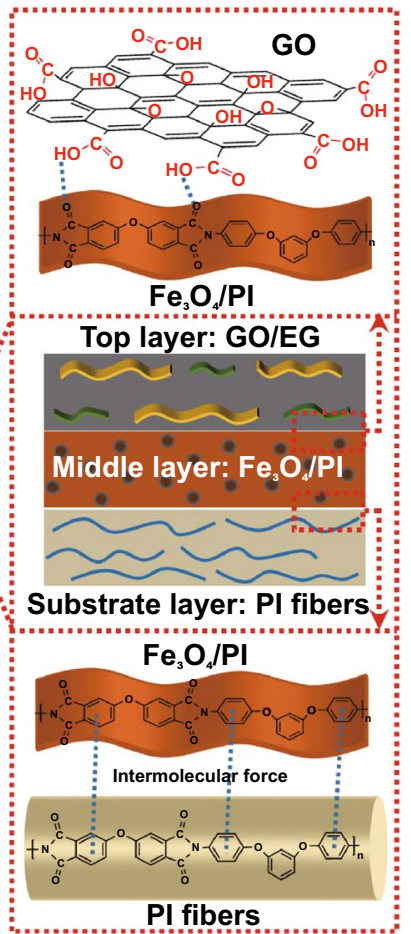

Fig. 1 Schematic diagram of the preparation process for PI composite films 
(Fig. S4e). As can be seen from Fig. 1, PI composite films have excellent flexibility and can be bent or folded into the shape of a paper crane without EG falling off, and there is no separation between layers. The main reason is that GO has good flexibility and high specific surface area to fix EG. The combination of the GO/EG layer and the $\mathrm{Fe}_{3} \mathrm{O}_{4} / \mathrm{PI}$ layer mainly relies on hydrogen bonding and $\pi-\pi$ interaction [38, 39]. The combination of the $\mathrm{Fe}_{3} \mathrm{O}_{4} / \mathrm{PI}$ layer and the PI fibers layer relies on the entanglement of PI molecular chains.

Figure 2 shows the morphologies of PI composite films. The overall cross section is shown in Fig. 2a. PI composite films have obvious hierarchical structure, and the overall thickness is about $245 \mu \mathrm{m}$. The layers of PI composite films are tightly connected to each other. The top layer of PI composite films is GO/EG film with thickness of about $70 \mu \mathrm{m}$, and the EG sheets arrange regularly and overlap each other (Fig. 2a, d). The main reason is that the addition of GO greatly improves the dispersibility of EG in water, and the directional flow of water presents an orderly structure during filtration. GO/EG films are compact, and the surface is smooth and has no defects such as wrinkles (Fig. 2b).
However, the GO/EG films without molding press have many fragments and gaps, and its surface is uneven (Fig. S2). The middle layer of PI composite films is $\mathrm{Fe}_{3} \mathrm{O}_{4} / \mathrm{PI}$ with thickness of about $16 \mu \mathrm{m}$. $\mathrm{Fe}_{3} \mathrm{O}_{4}$ is completely separated and coated by PI, and PI has an obvious orientation in the horizontal direction (Fig. 2e). The main reason is that the molecular chains of PAA spread in the horizontal direction due to the shearing force in the blade coating process, and the orientation characteristics of the molecular chains are fixed after solvent volatilization and thermal imidization [40]. The substrate layer of PI composite films is PI fibers with thickness of about $160 \mu \mathrm{m}$. PI fibers are fluffy and smooth (Fig. 2c, f).

By comparing the thermal conductivity coefficient of PI composite films with different components (Fig. S5), it can be found that the through-plane thermal conductivity coefficient $\left(\lambda_{\perp}\right)$ of PI composite films has no relationship with the amount of each layer, and remains at about $0.03 \mathrm{~W}$ $(\mathrm{m} \mathrm{K})^{-1}$. The in-plane thermal conductivity coefficient $(\lambda \|)$ of PI composite films is directly related to the amount of $\mathrm{GO} / \mathrm{EG}$, and is hardly affected by the amount of $\mathrm{Fe}_{3} \mathrm{O}_{4} / \mathrm{PI}$

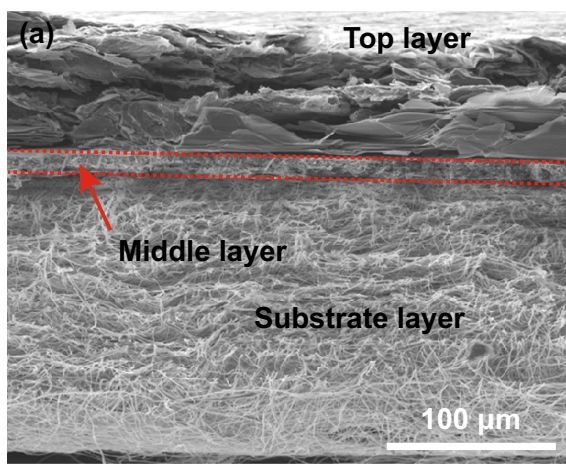

\section{(b)}
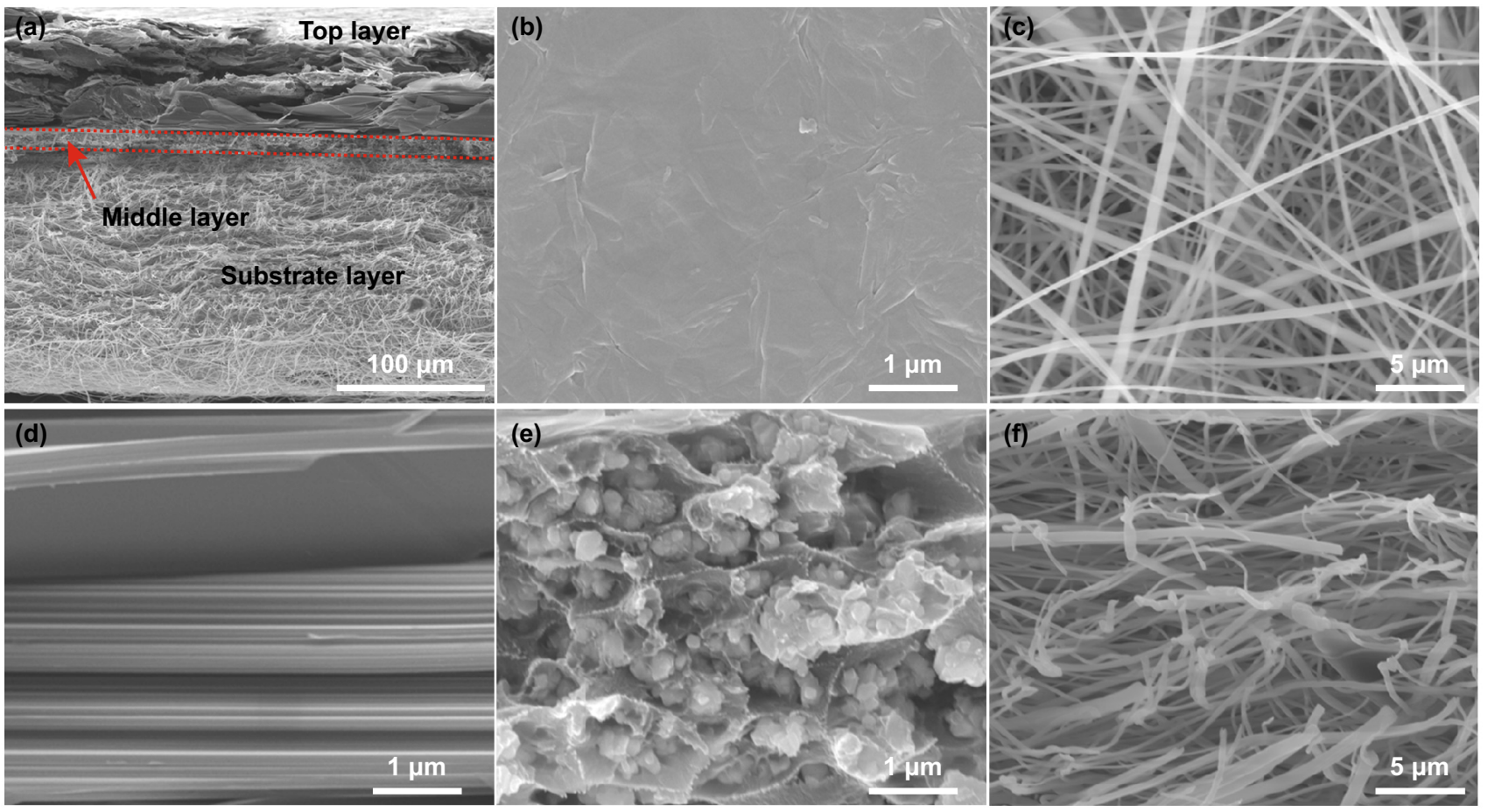

Fig. 2 SEM images of PI composite films. Side view (a), top view (b), bottom view (c), magnification of cross section of PI composite films $\left(\mathrm{GO} / \mathrm{EG}(\mathbf{d}), \mathrm{Fe}_{3} \mathrm{O}_{4} / \mathrm{PI}(\mathbf{e}), \mathrm{PI}\right.$ fibers (f)) 
and PI fibers. The $\lambda \|$ of PI composite films with fixed addition of $\mathrm{Fe}_{3} \mathrm{O}_{4} / \mathrm{PI}$ and PI fibers (39 and $25 \mathrm{mg}$, respectively) reaches maximum of $95.40 \mathrm{~W}(\mathrm{~m} \mathrm{~K})^{-1}$ when the amount of GO/EG is $61.0 \mathrm{wt} \%$ (Fig. 3a). The main reason is that the thickness of PI fibers layer of PI composite films is much thicker than that of the GO/EG layer and the $\mathrm{Fe}_{3} \mathrm{O}_{4} / \mathrm{PI}$ layer. The heat flow in the direction perpendicular to the plane of PI composite films will lose lots of energy when it is conducted through the PI fibers with low thermal conductivity, causing low $\lambda_{\perp}$ of PI composite films. When the amount of PI fibers in PI composite films is increased (that is, increasing the thickness of PI fibers mat), the conduction process of heat flow in the direction perpendicular to the PI composite films plane hardly changes, causing the $\lambda_{\perp}$ of PI composite films basically unchanged. In the direction of the PI composite films plane, GO/EG constructs perfect thermal conduction pathways and network, so that the heat flow can be quickly conducted under lower thermal resistance, giving PI composite films high $\lambda l l$. The more GO/EG, the more perfect of the thermal conduction pathways and network formed, which is conductive to the further enhancement in the $\lambda \| l$ of PI composite films. Based on the above results, this work fixes the thickness of GO/EG and PI fibrous layer of $70 \mu \mathrm{m}$ $(\sim 100 \mathrm{mg})$ and $160 \mu \mathrm{m}(\sim 25 \mathrm{mg})$ to make PI composite films possess the best thermal conductivity and thin thickness. The thermal conductivity of PI composite films varies with temperature as shown in Fig. 3b. It can be seen that both $\lambda \|$ and $\lambda_{\perp}$ of PI composite films increase slightly as the temperature increases. This is because the thermal motion of the molecules increases with the temperature increases [41].

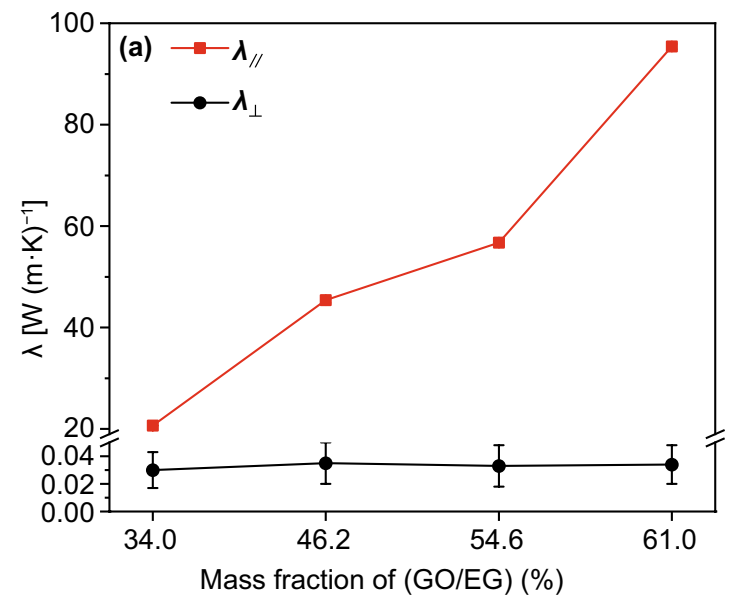

The thermal conductivity stability of PI composite films is evaluated by testing its thermal conductivity coefficient after bending (Fig. S6). The thermal conductivity ratio of PI composite films before and after bending is stable at $\sim 1$, indicating that its thermal conductivity does not change much after bending, showing good $\lambda$ stability.

Surface electrical conductivity ( $\sigma$ ) of PI composite films increases with the amount of GO/EG increases (Fig. 4a). The $\sigma$ of PI composite films with fixed addition of $\mathrm{Fe}_{3} \mathrm{O}_{4} /$ PI and PI fibers (39 and $25 \mathrm{mg}$, respectively) reaches the maximum of $230.0 \mathrm{~S} \mathrm{~cm}^{-1}$ when the amount of GO/EG is $61.0 \mathrm{wt} \%$. Connecting the PI composite films with the least and the largest amount of GO/EG into the lightemitting diode (LED) circuit (Voltage is $10 \mathrm{~V}$, LED lights are connected in parallel), the LED brightness of the latter is brighter (Fig. 4g, $g^{\prime}$ ), indicating lower resistance. However, the $\sigma$ of PI composite films has no relationship with the amount of $\mathrm{Fe}_{3} \mathrm{O}_{4} / \mathrm{PI}$ (Fig. S7), ascribing $\mathrm{Fe}_{3} \mathrm{O}_{4} / \mathrm{PI}$ located in the middle of PI composite films. The saturation magnetization of pure $\mathrm{Fe}_{3} \mathrm{O}_{4}$ and $\mathrm{Fe}_{3} \mathrm{O}_{4} / \mathrm{PI}(10$ wt. $\%$ ) is 74.8 and 7.1 emu g $^{-1}$, respectively (Fig. 4b). PI composite films can be easily attracted by the magnet (Fig. 4h, $\left.h^{\prime}\right)$. Under the condition of fixed amount of the top and substrate layer of PI composite films (GO/EG: $100 \mathrm{mg}$, PI fibers: $25 \mathrm{mg}$ ), the mass fraction of $\mathrm{Fe}_{3} \mathrm{O}_{4} / \mathrm{PI}$ in PI composite films increases with the proportion of $\mathrm{Fe}_{3} \mathrm{O}_{4}$ in $\mathrm{Fe}_{3} \mathrm{O}_{4} / \mathrm{PI}$ increases. From Fig. 4c, d, both the total EMI $\mathrm{SE}\left(\mathrm{EMI} \mathrm{SE}_{\mathrm{T}}\right.$ ) and the absorption efficiency $\left(\mathrm{EMI} \mathrm{SE}_{\mathrm{A}}\right)$ of the PI composite films increase with the percentage of $\mathrm{Fe}_{3} \mathrm{O}_{4} / \mathrm{PI}$ increases. When the middle layer of PI composite

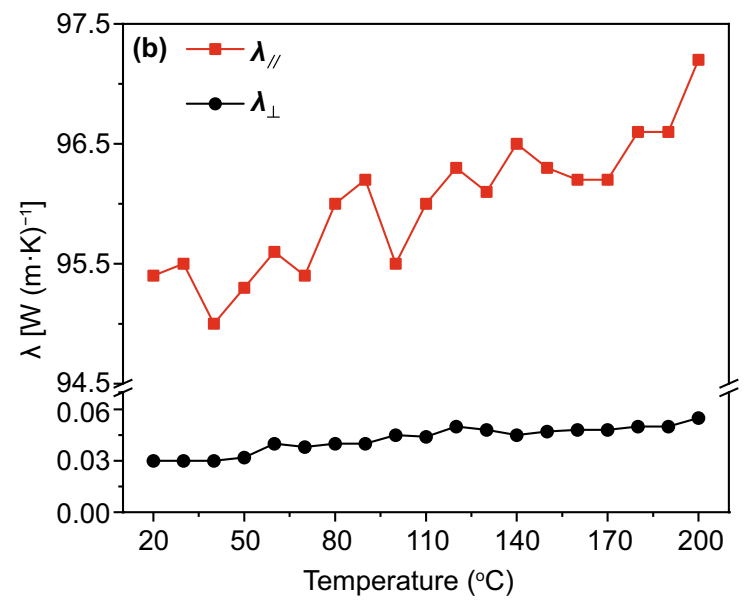

Fig. $3 \lambda$ of PI composite films with fixed addition of $\mathrm{Fe}_{3} \mathrm{O}_{4} / \mathrm{PI}$ and PI fibers (a), $\lambda$ of PI composite films contains 61.0 wt $\%$ of GO/EG change with temperature (b) 

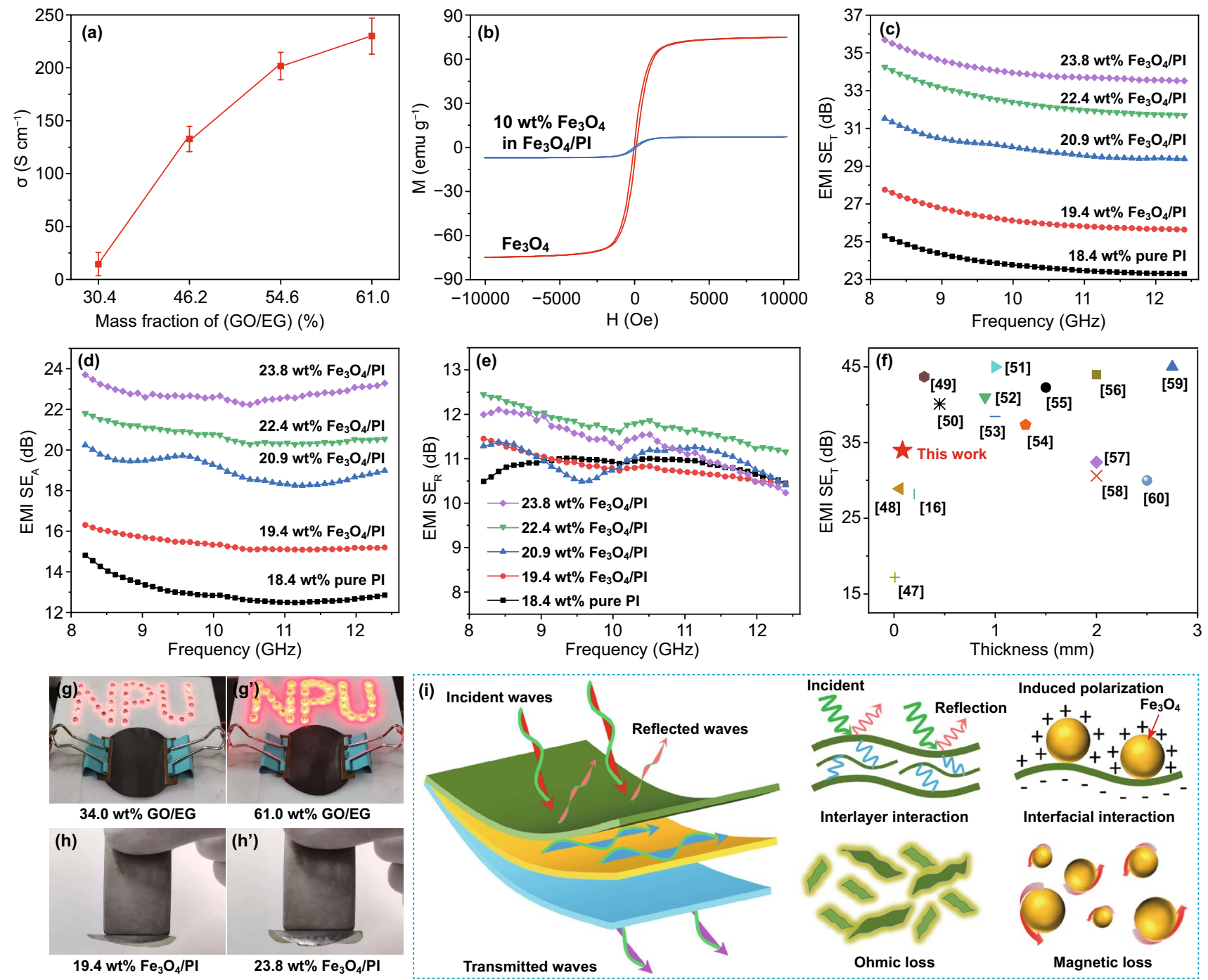

Fig. 4 Surface electrical conductivity of PI composite films with fixed addition of $\mathrm{Fe}_{3} \mathrm{O}_{4} / \mathrm{PI}$ and PI fibers (a), magnetization curves of Fe $\mathrm{O}_{4} /$ $\mathrm{PI}$ and $\mathrm{Fe}_{3} \mathrm{O}_{4}(\mathbf{b}), \mathrm{EMI} \mathrm{SE}_{\mathrm{T}}(\mathbf{c}), \mathrm{EMI} \mathrm{SE}_{\mathrm{A}}(\mathbf{d})$ and $\mathrm{EMI} \mathrm{SE}_{\mathrm{R}}(\mathbf{e})$ of PI composite films, summarization of EMI SE of PI composite films and other works (f), the digital photos showing electrical conductivity $\left(\mathbf{g}, \mathbf{g}^{\prime}\right)$ and magnetic properties $\left(\mathbf{h}, \mathbf{h}^{\prime}\right)$ of PI composite films, EMI shielding mechanism of PI composite films (i)

films is pure PI, the EMI $\mathrm{SE}_{\mathrm{T}}$ and $\mathrm{EMI} \mathrm{SE}_{\mathrm{A}}$ are the lowest, about 23.8 and $13.0 \mathrm{~dB}$, respectively, which increase to 34.0 and $22.8 \mathrm{~dB}$ when the middle layer is $\mathrm{Fe}_{3} \mathrm{O}_{4} / \mathrm{PI}$ and its amount is $23.8 \mathrm{wt} \%$. However, the reflection efficiency $\left(\mathrm{EMI} \mathrm{SE}_{\mathrm{R}}\right.$ ) of PI composite films is less affected by the amount of $\mathrm{Fe}_{3} \mathrm{O}_{4} / \mathrm{PI}$, and is basically maintained at about $11.0 \mathrm{~dB}$ (Fig. 4e). The main reason is that the EMI $\mathrm{SE}_{\mathrm{R}}$ is mainly related to the electrical conductivity [42]. In the case of fixed amount of GO/EG, the electrical conductivity of the PI composite films is basically unchanged, and the EMI $\mathrm{SE}_{\mathrm{R}}$ has little change. According to the structural characteristics of PI composite films, its
EMI shielding mechanism (Fig. 4i) may be concluded as follows. First, the incident electromagnetic wave excites high-frequency oscillating current on the surface of the GO/EG due to the impedance mismatch between GO/EG and the ambient space, reflecting part of the electromagnetic wave into the atmosphere. The remaining electromagnetic wave transmits to the inside of the GO/EG, and is reflected and scattered multiple times between the EG sheets [43]. In this process, each wavelet could interfere with each other and attenuate gradually. In addition, the highly oriented EG provides a large number of dipoles, which increases the loss ability of electromagnetic wave. 
After the electromagnetic wave enters the $\mathrm{Fe}_{3} \mathrm{O}_{4} / \mathrm{PI}$, the interface polarization is induced under the action of the external field, resulting in polarization loss due to the different electrical conductivity of the GO/EG and the $\mathrm{Fe}_{3} \mathrm{O}_{4} /$ PI. In addition, the $\mathrm{Fe}_{3} \mathrm{O}_{4}$ further loses electromagnetic wave in the form of natural resonance [44-46]. The EMI SE of PI composite films and other materials based on graphite derivatives and magnetic substances is summarized in Fig. 4f [16, 47-60]. It can be seen that the PI composite films prepared in this work has a relatively high specific efficiency (the ratio of EMI SE to the effective thickness of the material, and the effective thickness of PI composite films for EMI shielding is about $85 \mu \mathrm{m}$ ), which is about $400 \mathrm{~dB} \mathrm{~mm}^{-1}$.

PI composite films shows good electric-heating properties based on the Joule effect, which could be used in the fields including defogging and defrosting. Fast electric-heating response and excellent thermal conductivity can ensure rapid generation and conduction of a large amount of heat, which can reduce energy waste. The electric-heating results of PI composite films (GO/EG: $61.0 \mathrm{wt} \%, \mathrm{Fe}_{3} \mathrm{O}_{4} / \mathrm{PI}: 23.8 \mathrm{wt} \%$,
PI fibers: $15.2 \mathrm{wt} \%$ ) with the best thermal conductivity and EMI shielding performance are shown in Fig. 5. Figure 5a, $\mathrm{b}$ shows the time-temperature relationship of PI composite films with different voltages. The temperature of the top and substrate layer at stable condition increases with the voltage increases, indicating that more Joule heat is generated. The temperature of the substrate layer of PI composite films is lower than the top layer in the condition of the same voltage. For example, the temperature of the top layer of the PI composite films with voltage of $10 \mathrm{~V}$ is about $163^{\circ} \mathrm{C}$, while substrate layer is about $134^{\circ} \mathrm{C}$. The main reason is that the top layer of PI composite films is conductive, which generates heat due to the Joule effect. The substrate layer of PI composite films is insulated, which cannot generate Joule heat. The heat of the top layer of PI composite films is partially lost in the process of conducting to the substrate layer, and the low through-plane thermal conductivity of PI composite films hinders the heat diffusion through the layers, resulting in the lower temperature of substrate layer. In order to fully understand the electric-heating mechanism, thermodynamic analysis is conducted. Based on the principle
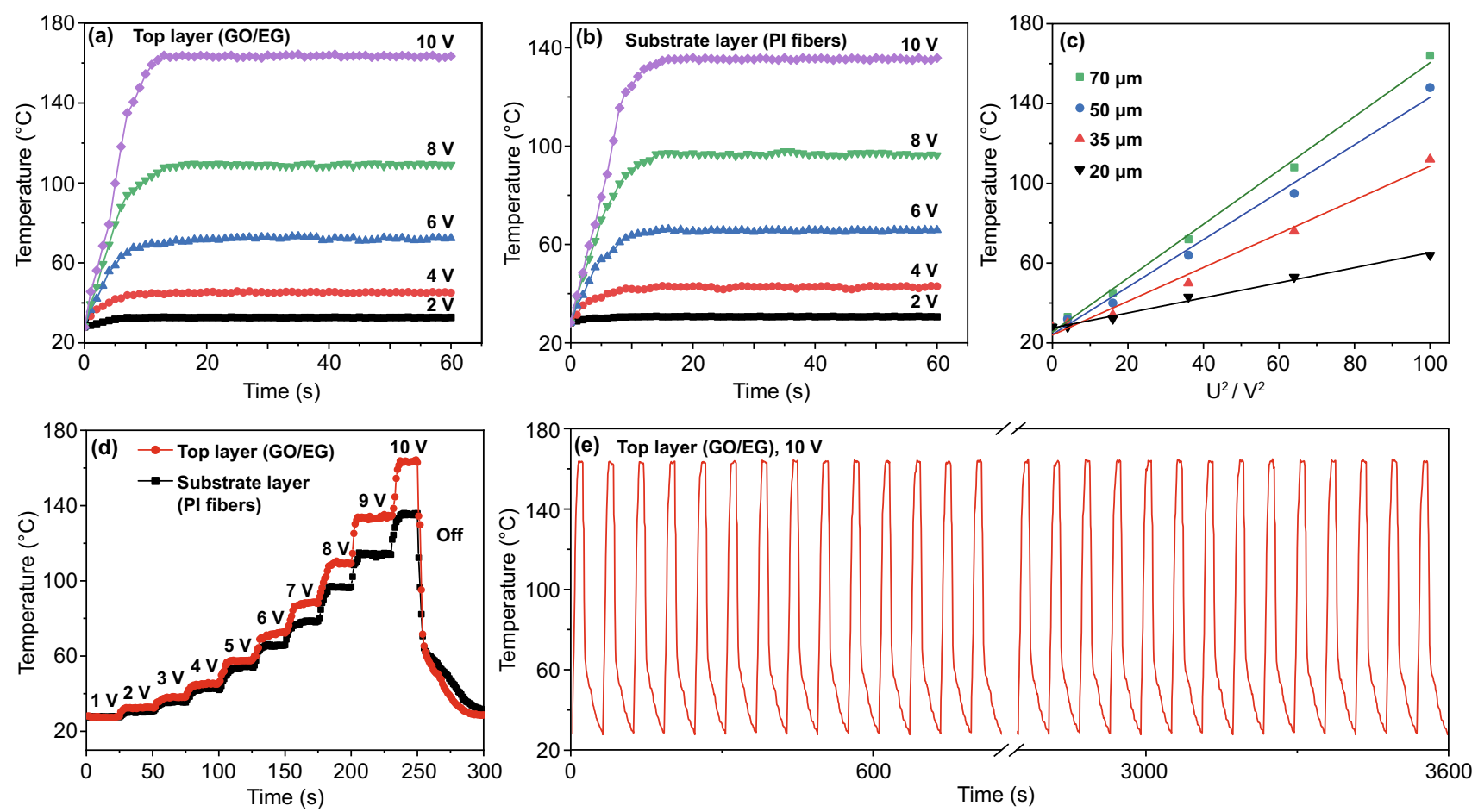

Fig. 5 Electric-heating property of PI composite films. The time-temperature relationship of PI composite films with different voltages (a, b), linear fitting of experimental temperature versus $U^{2}(\mathbf{c})$, surface temperatures of the PI composite films upon gradiently changed voltages (d), electric-heating stability of the PI composite films upon repeated supplied voltages (e) 
of energy conservation, the consumed electrical energy is equal to the generated thermal energy when the temperature of PI composite films is stable. The surface temperature of PI composite films follows Eq. 1 [61]:

$T_{\mathrm{s}}=T_{0}+\frac{U^{2}}{R h A}$

where $T_{0}$ and $T_{\mathrm{s}}$ are the initial and stable temperature of the PI composite films, $U$ is the voltage applied to the PI composite films, $h$ is the convective heat transfer coefficient, and $R$ and $A$ are the resistance and surface area of the PI composite films, respectively.

The relationship between the temperature of the top layer of the PI composite films and the square of the applied voltage is shown in Fig. 5c. It can be seen that $T_{\mathrm{s}}$ has a linear relationship with $U^{2}$, and the slope is $1 / R$. This indicates that the thicker the GO/EG, the higher perfection of the conductive networks and the lower the resistance.

Figure $5 \mathrm{~d}$ shows the relationship between the surface temperature of the PI composite films and the gradient voltage.
Surface temperature of the PI composite films stabilized quickly when the voltage gradually increases with the gradient of $1 \mathrm{~V}$, and the response time is about $5 \mathrm{~s}$. The temperature of the top layer of the PI composite films is always higher than the substrate layer. The higher the voltage, the greater the temperature differences. When the voltage is cut off, the surface temperature of the PI composite films decreases rapidly, and the cooling rate slows down after $5 \mathrm{~s}$. To evaluate the reliability and stability of the electricheating property, periodic constant voltage is applied to the PI composite films (The voltage is $10 \mathrm{~V}$. Each cycle lasts $60 \mathrm{~s}$, the voltage is applied for the first $20 \mathrm{~s}$, and then cut off), the result is shown in Fig. 5e. The temperature of the top layer of PI composite films is stable at about $163{ }^{\circ} \mathrm{C}$. At the beginning of each cycle, the temperature rises rapidly to be stable, and then the temperature drops rapidly to about $60{ }^{\circ} \mathrm{C}$ and then slowly decreases when the voltage is cut off. The process lasts for $6 \mathrm{~h}$, and the temperature changes of PI composite films has good repeatability.

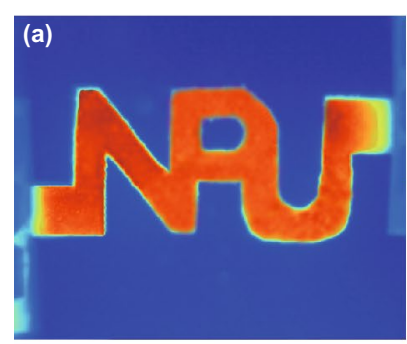

$10 \mathrm{~V}$
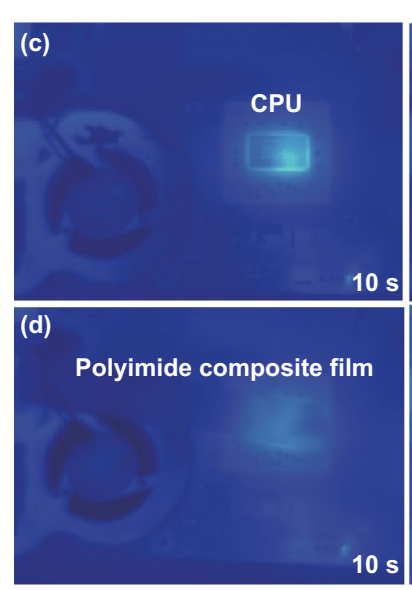

IR images of bare CPU and CPU integrated with HMPF
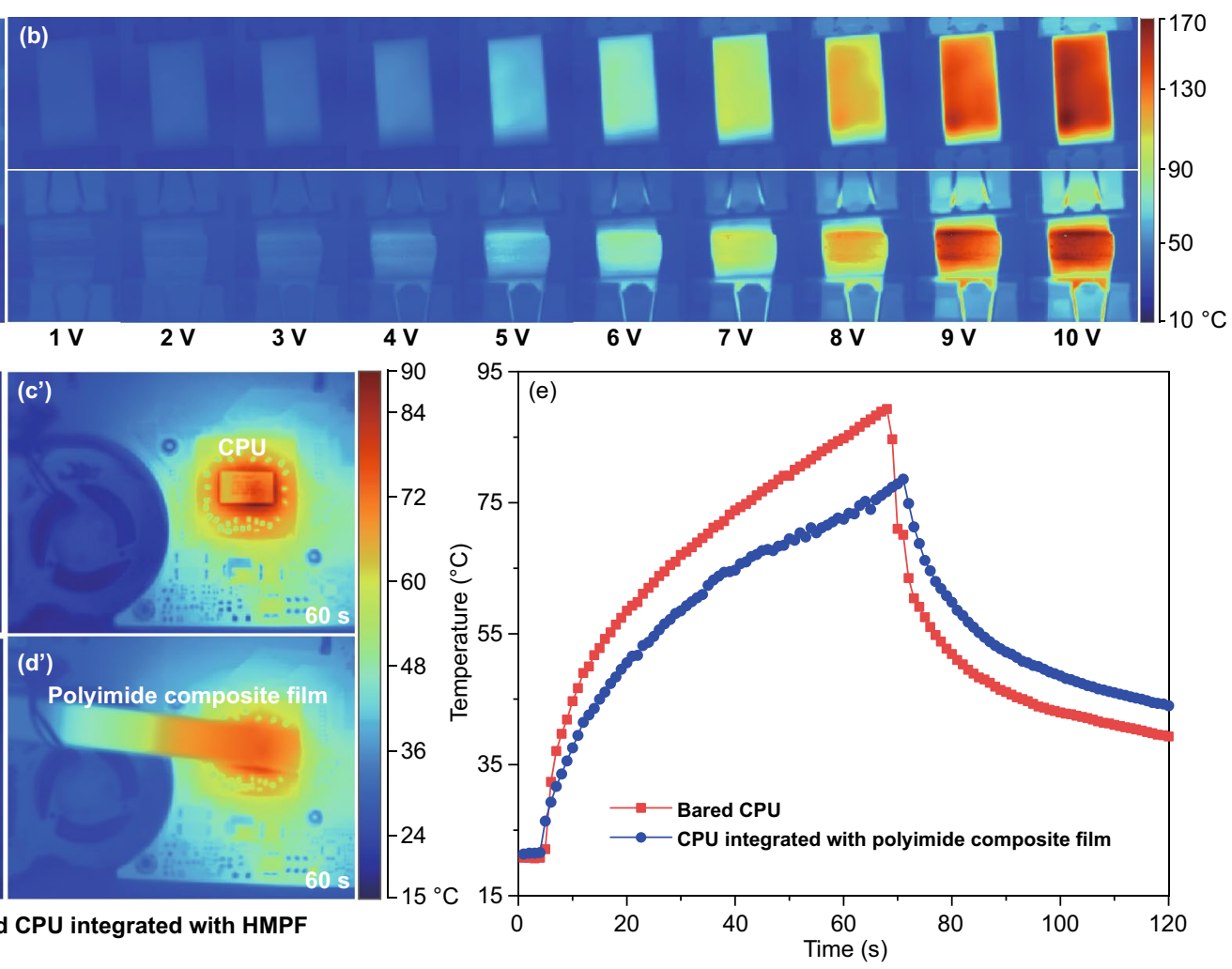

Fig. 6 Infrared thermal images of PI composite films. IR images of PI composite films with the shape of "NPU" at 10 V voltage (a), IR images of the PI composite films upon bending and stretching (b), IR images of bare CPU (c, $\mathbf{c}^{\prime}$ ) and integrated with PI composite films (d, d'), the working temperature of CPU (e) 

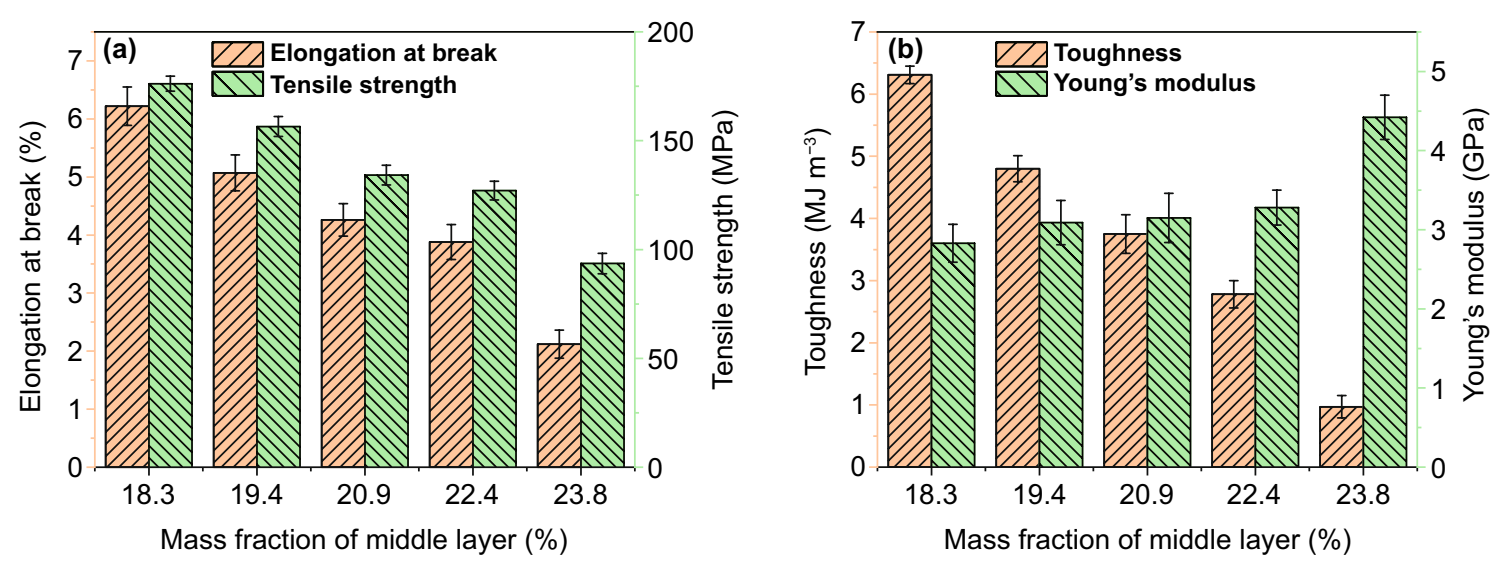

Fig. 7 Tensile strength and elongation at break (a), toughness and Young's modulus (b) of PI composite film

Infrared thermal (IR) images can clearly reflect the thermal performance of PI composite films. Figure 6a shows the electric-heating performance (applied voltage of $10 \mathrm{~V}$ ) of the PI composite films with a shape of "NPU." The temperature distribution in the PI composite films is uniform, indicating the PI composite films has uniform thermal/electrical conduction networks. Figure $6 \mathrm{~b}$ shows the IR images of the PI composite films upon bending and stretching. The uniform temperature distribution during the bending indicates the bending has not destroyed the thermal/electrical conduction networks. The actual heat dissipation of PI composite films is characterized by the temperature change of the central processing unit (CPU) of computer. The temperature of the bared CPU (Fig. S8a) rises rapidly during working (Fig. 6c, $\mathrm{c}^{\prime}$ ), the surface temperature rises to $90^{\circ} \mathrm{C}$ in $70 \mathrm{~s}$, and slowly drops to $40{ }^{\circ} \mathrm{C}$ after the power is turned off (Fig. 6e). The surface temperature of the CPU integrated with PI composite films (Fig. S8b) rises slowly (Fig. 6d, d'), which is $78^{\circ} \mathrm{C}$ at $70 \mathrm{~s}\left(12{ }^{\circ} \mathrm{C}\right.$ lower than that of the bare CPU), and it drops slowly after turning off the power. It shows that the PI composite films can effectively reduce the working temperature of $\mathrm{CPU}$, and preventing the temperature of $\mathrm{CPU}$ from changing sharply.

Figure 7 shows the tensile strength, elongation at break, toughness and Young's modulus obtained from the stress-strain curve of PI composite films (Fig. S9). Tensile strength, elongation at break and toughness of PI composite films decrease with the increase in the amount of $\mathrm{Fe}_{3} \mathrm{O}_{4} / \mathrm{PI}$, while the Young's modulus increases when the amount of $\mathrm{Fe}_{3} \mathrm{O}_{4} / \mathrm{PI}$ increases. When $\mathrm{Fe}_{3} \mathrm{O}_{4} / \mathrm{PI}$ amount is $23.8 \mathrm{wt} \%$, the tensile strength, elongation at break, toughness and Young's modulus of PI composite films are about 93.6 MPa, 2.12\%, $0.97 \mathrm{MJ} \mathrm{m}^{-3}$ and $4.42 \mathrm{GPa}$, respectively. The increase in the amount of $\mathrm{Fe}_{3} \mathrm{O}_{4} / \mathrm{PI}$ in PI composite films is mainly due to the increase in the amount of $\mathrm{Fe}_{3} \mathrm{O}_{4}$ in $\mathrm{Fe}_{3} \mathrm{O}_{4} / \mathrm{PI}$, which increases the defects of PI [62].

\section{Conclusion}

In this work, the obtained hierarchically multifunctional PI composite films have a three-layer structure, the top layer is $\mathrm{GO} / \mathrm{EG}$ for thermal conduction and EMI shielding, the middle layer is $\mathrm{Fe}_{3} \mathrm{O}_{4} / \mathrm{PI}$ for enhancing the EMI shielding effectiveness, the substrate layer is PI fibers for improving the mechanical property. The top, middle and substrate layers of PI composite films are tightly bonded. PI composite films with $61.0 \mathrm{wt} \%$ and $23.8 \mathrm{wt} \%$ of $\mathrm{GO} / \mathrm{EG}$ and $\mathrm{Fe}_{3} \mathrm{O}_{4} /$ PI, respectively, possess the highest $\lambda \|\left(95.40 \mathrm{~W}\left(\mathrm{~m} \mathrm{~K}^{-1}\right)\right.$, EMI SE (34.0 dB), excellent tensile strength (93.6 MPa) and fast electric-heating response ( $5 \mathrm{~s}$, voltage gradient is $1 \mathrm{~V}$ ). Taking the CPU as the actual heat dissipation scenario verifies that PI composite films has broad application prospects in the field of light and miniaturized electronic equipment.

Acknowledgements The authors are grateful for the support and funding from National Natural Science Foundation of China (51773169 and 51973173); Technical Basis Scientific Research Project (Highly Thermally Conductive Nonmetal Materials); Guangdong Basic and Applied Basic Research Foundation (2019B1515120093); Natural Science Basic Research Plan for Distinguished Young Scholars in Shaanxi Province of China (2019JC11). Y.Q. Guo thanks for the Innovation Foundation for Doctor Dissertation of Northwestern Polytechnical University (CX202055). 
This work is also financially supported by Polymer Electromagnetic Functional Materials Innovation Team of Shaanxi Sanqin Scholars.

Funding Open access funding provided by Shanghai Jiao Tong University.

Open Access This article is licensed under a Creative Commons Attribution 4.0 International License, which permits use, sharing, adaptation, distribution and reproduction in any medium or format, as long as you give appropriate credit to the original author(s) and the source, provide a link to the Creative Commons licence, and indicate if changes were made. The images or other third party material in this article are included in the article's Creative Commons licence, unless indicated otherwise in a credit line to the material. If material is not included in the article's Creative Commons licence and your intended use is not permitted by statutory regulation or exceeds the permitted use, you will need to obtain permission directly from the copyright holder. To view a copy of this licence, visit http://creativecommons.org/licenses/by/4.0/.

Supplementary Information The online version contains supplementary material available at https://doi.org/10.1007/ s40820-021-00767-4.

\section{References}

1. M. Shtein, R. Nadiv, M. Buzaglo, O. Regev, Graphenebased hybrid composites for efficient thermal management of electronic devices. ACS Appl. Mater. Interfaces 7(42), 23725-23730 (2015). https://doi.org/10.1021/acsami.5b078 66

2. S.J. Kang, H. Hong, C. Jeong, J.S. Lee, H. Ryu et al., Avoiding heating interference and guided thermal conduction in stretchable devices using thermal conductive composite islands. Nano Res. 14(9), 3253-3259 (2021). https://doi.org/10.1007/ s12274-021-3400-5

3. X. Qian, J. Zhou, G. Chen, Phonon-engineered extreme thermal conductivity materials. Nat. Mater. 20, 1188-1202 (2021). https://doi.org/10.1038/s41563-021-00918-3

4. L. Yu, D. Yang, Q. Wei, L. Zhang, Constructing of strawberrylike core-shell structured $\mathrm{Al}_{2} \mathrm{O}_{3}$ nanoparticles for improving thermal conductivity of nitrile butadiene rubber composites. Compos. Sci. Technol. 209, 108786 (2021). https://doi.org/10. 1016/j.compscitech.2021.108786

5. J. Gu, K. Ruan, Breaking through bottlenecks for thermally conductive polymer composites: a perspective for intrinsic thermal conductivity, interfacial thermal resistance and theoretics. Nano-Micro Lett. 13, 110 (2021). https://doi.org/10. 1007/s40820-021-00640-4

6. F. Jiang, X. Cui, N. Song, L. Shi, P. Ding, Synergistic effect of functionalized graphene/boron nitride on the thermal conductivity of polystyrene composites. Compos. Commun. 20, 100350 (2020). https://doi.org/10.1016/j.coco.2020.04.016

7. H. Yu, Y. Feng, C. Chen, Z. Zhang, Y. Cai et al., Thermally conductive, self-healing, and elastic polyimide@vertically aligned carbon nanotubes composite as smart thermal interface material. Carbon 179, 348-357 (2021). https://doi.org/10. 1016/j.carbon.2021.04.055

8. P. Song, B. Liu, C. Liang, K. Ruan, H. Qiu et al., Lightweight, flexible cellulose-derived carbon aerogel@reduced graphene oxide/PDMS composites with outstanding EMI shielding performances and excellent thermal conductivities. Nano-Micro Lett. 13, 91 (2021). https://doi.org/10.1007/ s40820-021-00624-4

9. D. Wang, Y. Lin, D. Hu, P. Jiang, X. Huang, Multifunctional 3D-MXene/PDMS nanocomposites for electrical, thermal and triboelectric applications. Compos. Part A Appl. Sci. Manuf. 130, 105754 (2020). https://doi.org/10.1016/j.compositesa. 2019.105754

10. C. Tan, Z. Dong, Y. Li, H. Zhao, X. Huang et al., A high performance wearable strain sensor with advanced thermal management for motion monitoring. Nat. Commun. 11, 3530 (2020). https://doi.org/10.1038/s41467-020-17301-6

11. K. Ruan, X. Zhong, X. Shi, J. Dang, J. Gu, Liquid crystal epoxy resins with high intrinsic thermal conductivities and their composites: a mini-review. Mater. Today Phys. 20, 100456 (2021). https://doi.org/10.1016/j.mtphys.2021.100456

12. F. Zhang, Y. Feng, W. Feng, Three-dimensional interconnected networks for thermally conductive polymer composites: design, preparation, properties, and mechanisms. Mater. Sci. Eng. R Rep. 142, 100580 (2020). https://doi.org/ 10.1016/j.mser.2020.100580

13. W. Yu, C. Liu, S. Fan, Advances of CNT-based systems in thermal management. Nano Res. 14(8), 2471-2490 (2021). https://doi.org/10.1007/s12274-020-3255-1

14. N. Kozak, L. Matzui, L. Vovchenko, L. Kosyanchuk, V. Oliynyk et al., Influence of coordination complexes of transition metals on EMI-shielding properties and permeability of polymer blend/carbon nanotube/nickel composites. Compos. Sci. Technol. 200, 108420 (2020). https://doi.org/10.1016/j. compscitech.2020.108420

15. L.X. Liu, W. Chen, H.B. Zhang, Q.W. Wang, F. Guan et al., Flexible and multifunctional silk textiles with biomimetic leaf-like MXene/silver nanowire nanostructures for electromagnetic interference shielding, humidity monitoring, and self-derived hydrophobicity. Adv. Funct. Mater. 29(44), 1905197 (2019). https://doi.org/10.1002/adfm.201905197

16. Y.Y. Wang, W.J. Sun, D.X. Yan, K. Dai, Z.M. Li, Ultralight carbon nanotube/graphene/polyimide foam with heterogeneous interfaces for efficient electromagnetic interference shielding and electromagnetic wave absorption. Carbon 176, 118-125 (2021). https://doi.org/10.1016/j.carbon.2020.12. 028

17. R. Yang, X. Gui, L. Yao, Q. Hu, L. Yang et al., Ultrathin, lightweight, and flexible CNT buckypaper enhanced using MXenes for electromagnetic interference shielding. Nano-Micro Lett. 13, 66 (2021). https://doi.org/10.1007/s40820-021-00597-4

18. J. Yang, X. Liao, G. Wang, J. Chen, P. Song et al., Heterogeneous silicon rubber composite foam with gradient porous structure for highly absorbed ultra-efficient electromagnetic 
interference shielding. Compos. Sci. Technol. 206, 108663 (2021). https://doi.org/10.1016/j.compscitech.2021.108663

19. W. Liu, T. Yao, K. Jia, J. Gu, D. Wang et al., Flexible and thermal conducting multi-walled carbon nanotubes/waterborne polyurethane composite film from in situ polymerization for efficient electromagnetic interference shielding. J. Mater. Sci. Mater. Electron. 32(4), 4393-4403 (2021). https://doi.org/10. 1007/s10854-020-05182-w

20. W. Yang, Z. Zhao, K. Wu, R. Huang, T. Liu et al., Ultrathin flexible reduced graphene oxide/cellulose nanofiber composite films with strongly anisotropic thermal conductivity and efficient electromagnetic interference shielding. J. Mater. Chem. C 5(15), 3748-3756 (2017). https://doi.org/10.1039/c7tc0 $0400 \mathrm{a}$

21. K. Ruan, Y. Guo, C. Lu, X. Shi, T. Ma et al., Significant reduction of interfacial thermal resistance and phonon scattering in graphene/polyimide thermally conductive composite films for thermal. Research 2021, 8438614 (2021). https://doi.org/10. 34133/2021/8438614

22. K. Ruan, Y. Guo, J. Gu, Liquid crystalline polyimide films with high intrinsic thermal conductivities and robust toughness. Macromolecules 54(10), 4934-4944 (2021). https://doi. org/10.1021/acs.macromol.1c00686

23. H. Liu, R. Fu, X. Su, B. Wu, H. Wang et al., Electrical insulating MXene/PDMS/BN composite with enhanced thermal conductivity for electromagnetic shielding application. Compos. Commun. 23, 100593 (2021). https://doi.org/10.1016/j.coco. 2020.100593

24. W. Wang, X. Ma, Y. Shao, X. Qi, J. Yang et al., Flexible, multifunctional, and thermally conductive nylon/graphene nanoplatelet composite papers with excellent EMI shielding performance, improved hydrophobicity and flame resistance. J. Mater. Chem. A 9(8), 5033-5044 (2021). https://doi.org/10. 1039/D0TA11040J

25. L. Li, Z. Ma, P. Xu, B. Zhou, Q. Li et al., Flexible and alternant-layered cellulose nanofiber/graphene film with superior thermal conductivity and efficient electromagnetic interference shielding. Compos. Part A Appl. Sci. Manuf. 139, 106134 (2020). https://doi.org/10.1016/j.compositesa.2020.106134

26. Q. Wei, D. Yang, L. Yu, L. Zhang, Grafting of epoxidized natural rubber chains with BN platelets to obtain flexible and thermally conductive papers. Compos. Sci. Technol. 212, 108881 (2021). https://doi.org/10.1016/j.compscitech.2021. 108881

27. Y. Chen, L. Sui, H. Fang, C. Ding, Z. Li et al., Superior mechanical enhancement of epoxy composites reinforced by polyimide nanofibers via a vacuum-assisted hot-pressing. Compos. Sci. Technol. 174, 20-26 (2019). https://doi.org/10. 1016/j.compscitech.2019.02.012

28. C. Liang, P. Song, H. Qiu, Y. Huangfu, Y. Lu et al., Superior electromagnetic interference shielding performances of epoxy composites by introducing highly aligned reduced graphene oxide films. Compos. Part A Appl. Sci. Manuf. 124, 105512 (2019). https://doi.org/10.1016/j.compositesa.2019.105512

29. B. Shen, W. Zhai, W. Zheng, Ultrathin flexible graphene film: an excellent thermal conducting material with efficient EMI shielding. Adv. Funct. Mater. 24(28), 4542-4548 (2014). https://doi.org/10.1002/adfm.201400079

30. Y. Liu, J. Zeng, D. Han, K. Wu, B. Yu et al., Graphene enhanced flexible expanded graphite film with high electric, thermal conductivities and EMI shielding at low content. Carbon 133, 435-445 (2018). https://doi.org/10.1016/j.carbon. 2018.03.047

31. W.B. Zhang, Z.X. Zhang, J.H. Yang, T. Huang, N. Zhang et al., Largely enhanced thermal conductivity of poly(vinylidene fluoride)/carbon nanotube composites achieved by adding graphene oxide. Carbon 90, 242-254 (2015). https://doi.org/ 10.1016/j.carbon.2015.04.040

32. L. Wang, Z. Ma, Y. Zhang, L. Chen, D. Cao et al., Polymerbased EMI shielding composites with $3 \mathrm{D}$ conductive networks: a mini-review. SusMat 1(3), 413-431 (2021). https:// doi.org/10.1002/sus2.21

33. Q. Liu, Q. Cao, H. Bi, C. Liang, K. Yuan et al., CoNi@ $\mathrm{SiO}_{2} @$ $\mathrm{TiO}_{2}$ and $\mathrm{CoNi} @ \mathrm{Air} @ \mathrm{TiO}_{2}$ microspheres with strong wideband microwave absorption. Adv. Mater. 28(3), 486-490 (2016). https://doi.org/10.1002/adma.201503149

34. Y. Zhang, K. Ruan, J. Gu, Flexible sandwich-structured electromagnetic interference shielding nanocomposite films with excellent thermal conductivities. Small 17(42), 2101951 (2021). https://doi.org/10.1002/smll.202101951

35. Y. Liu, B. Qu, X. Wu, Y. Tian, K. Wu et al., Utilizing ammonium persulfate assisted expansion to fabricate flexible expanded graphite films with excellent thermal conductivity by introducing wrinkles. Carbon 153, 565-574 (2019). https://doi.org/10.1016/j.carbon.2019.07.079

36. Y. Guo, K. Ruan, J. Gu, Controllable thermal conductivity in composites by constructing thermal conduction networks. Mater. Today Phys. 20, 100449 (2021). https://doi.org/10. 1016/j.mtphys.2021.100449

37. B. Qi, X. He, G. Zeng, Y. Pan, G. Li et al., Strict molecular sieving over electrodeposited 2D-interspacing-narrowed graphene oxide membranes. Nat. Commun. 8(1), 825 (2017). https://doi.org/10.1038/s41467-017-00990-x

38. J.Y. Wang, S.Y. Yang, Y.L. Huang, H.W. Tien, W.K. Chin et al., Preparation and properties of graphene oxide/polyimide composite films with low dielectric constant and ultrahigh strength via in situpolymerization. J. Mater. Chem. 21(35), 13569-13575 (2011). https://doi.org/10.1039/ C1JM11766A

39. N. Song, D. Jiao, S. Cui, X. Hou, P. Ding et al., Highly anisotropic thermal conductivity of layer-by-layer assembled nanofibrillated cellulose/graphene nanosheets hybrid films for thermal management. ACS Appl. Mater. Interfaces 9(3), 2924-2932 (2017). https://doi.org/10.1021/acsami.6b11979

40. M. Niwa, H. Kawakami, T. Kanamori, T. Shinbo, A. Kaito et al., Gas separation of asymmetric 6FDA polyimide membrane with oriented surface skin layer. Macromolecules 34(26), 9039-9044 (2001). https://doi.org/10.1021/ma011 3778

41. H.C. Yuan, C.Y. Lee, N.H. Tai, Extremely high thermal conductivity of nanodiamond-polydopamine/thin-layer graphene 
composite films. Compos. Sci. Technol. 167, 313-322 (2018). https://doi.org/10.1016/j.compscitech.2018.08.010

42. Z. Wang, B. Mao, Q. Wang, J. Yu, J. Dai et al., Ultrahigh conductive copper/large flake size graphene heterostructure thin-film with remarkable electromagnetic interference shielding effectiveness. Small 14(20), 1704332 (2018). https://doi. org/10.1002/smll.201704332

43. Z. Wang, P. Li, R. Song, W. Qian, H. Zhou et al., High conductive graphene assembled films with porous micro-structure for freestanding and ultra-low power strain sensors. Sci. Bull. 65(16), 1363-1370 (2020). https://doi.org/10.1016/j.scib. 2020.05.002

44. H. Fang, H. Guo, Y. Hu, Y. Ren, P.C. Hsu et al., In-situ grown hollow $\mathrm{Fe}_{3} \mathrm{O}_{4}$ onto graphene foam nanocomposites with high EMI shielding effectiveness and thermal conductivity. Compos. Sci. Technol. 188, 107975 (2020). https://doi.org/10. 1016/j.compscitech.2019.107975

45. Z. Deng, P. Tang, X. Wu, H.B. Zhang, Z.Z. Yu, Superelastic, ultralight, and conductive $\mathrm{Ti}_{3} \mathrm{C}_{2} \mathrm{~T}_{\mathrm{x}}$ MXene/acidified carbon nanotube anisotropic aerogels for electromagnetic interference shielding. ACS Appl. Mater. Interfaces 13(17), 20539-20547 (2021). https://doi.org/10.1021/acsami.1c02059

46. A. Gupta, V. Choudhary, Electromagnetic interference shielding behavior of poly(trimethylene terephthalate)/multi-walled carbon nanotube composites. Compos. Sci. Technol. 71(13), 1563-1568 (2011). https://doi.org/10.1016/j.compscitech. 2011.06.014

47. P. Cataldi, D.G. Papageorgiou, G. Pinter, A.V. Kretinin, W.W. Sampson et al., Graphene-polyurethane coatings for deformable conductors and electromagnetic interference shielding. Adv. Electron. Mater. 6(9), 2000429 (2020). https://doi.org/ 10.1002/aelm.202000900

48. J. Hong, P. Xu, H. Xia, Z. Xu, Q.Q. Ni, Electromagnetic interference shielding anisotropy enhanced by CFRP laminated structures. Compos. Sci. Technol. 203, 108616 (2021). https:// doi.org/10.1016/j.compscitech.2020.108616

49. B. Zhao, S. Zeng, X. Li, X. Guo, Z. Bai et al., Flexible PVDF/ carbon materials/Ni composite films maintaining strong electromagnetic wave shielding under cyclic microwave irradiation. J. Mater. Chem. C 8(2), 500-509 (2020). https://doi.org/ 10.1039/C9TC05462F

50. Y. Wang, H.K. Peng, T.T. Li, B.C. Shiu, X. Zhang et al., Lightweight, flexible and superhydrophobic conductive composite films based on layer-by-layer self-assembly for high-performance electromagnetic interference shielding. Compos. Part A Appl. Sci. Manuf. 141, 106199 (2021). https://doi.org/10. 1016/j.compositesa.2020.106199

51. S. Das, S. Sharma, T. Yokozeki, S. Dhakate, Conductive layerbased multifunctional structural composites for electromagnetic interference shielding. Compos. Struct. 261, 113293 (2021). https://doi.org/10.1016/j.compstruct.2020.113293

52. K.Y. Chen, S. Gupta, N.H. Tai, Reduced graphene oxide/ $/ \mathrm{Fe}_{2} \mathrm{O}_{3}$ hollow microspheres coated sponges for flexible electromagnetic interference shielding composites. Compos. Commun.
23, 100572 (2021). https://doi.org/10.1016/j.coco.2020. 100572

53. S. Mondal, S. Ganguly, M. Rahaman, A. Aldalbahi, T.K. Chaki et al., A strategy to achieve enhanced electromagnetic interference shielding at low concentration with a new generation of conductive carbon black in a chlorinated polyethylene elastomeric matrix. Phys. Chem. Chem. Phys. 18(35), 24591-24599 (2016). https://doi.org/10.1039/C6CP04274K

54. Y. Zhan, M. Oliviero, J. Wang, A. Sorrentino, G.G. Buonocore et al., Enhancing the EMI shielding of natural rubber-based supercritical $\mathrm{CO}_{2}$ foams by exploiting their porous morphology and CNT segregated networks. Nanoscale 11(3), 10111020 (2019). https://doi.org/10.1039/C8NR07351A

55. Q. Yuchang, W. Qinlong, L. Fa, Z. Wancheng, Z. Dongmei, Graphene nanosheets/BaTiO ${ }_{3}$ ceramics as highly efficient electromagnetic interference shielding materials in the X-band. J. Mater. Chem. C 4(2), 371-375 (2016). https://doi.org/10.1039/ C5TC03035H

56. W.L. Song, M.S. Cao, B. Wen, Z.L. Hou, J. Cheng et al., Synthesis of zinc oxide particles coated multiwalled carbon nanotubes: dielectric properties, electromagnetic interference shielding and microwave absorption. Mater. Res. Bull. 47(7), 1747-1754 (2012). https://doi.org/10.1016/j.materresbull. 2012.03.045

57. J. Jing, Y. Xiong, S. Shi, H. Pei, Y. Chen et al., Facile fabrication of lightweight porous FDM-Printed polyethylene/graphene nanocomposites with enhanced interfacial strength for electromagnetic interference shielding. Compos. Sci. Technol. 207, 108732 (2021). https://doi.org/10.1016/j.compscitech. 2021.108732

58. T. Zheng, S.M. Sabet, S. Pilla, Polydopamine coating improves electromagnetic interference shielding of delignified woodderived carbon scaffold. J. Mater. Sci. 56(18), 10915-10925 (2021). https://doi.org/10.1007/s10853-021-06007-9

59. R. Kumar, S.R. Dhakate, T. Gupta, P. Saini, B.P. Singh et al., Effective improvement of the properties of light weight carbon foam by decoration with multi-wall carbon nanotubes. J. Mater. Chem. A 1(18), 5727-5735 (2013). https://doi.org/10. 1039/C3TA10604G

60. O. Lozitsky, L. Vovchenko, L. Matzui, V. Oliynyk, V. Zagorodnii, Microwave properties of epoxy composites with mixed filler carbon nanotubes/BaTiO 3 . Appl. Nanosci. 10(8), 27592767 (2020). https://doi.org/10.1007/s13204-020-01402-1

61. Z. Ma, S. Kang, J. Ma, L. Shao, A. Wei et al., High-performance and rapid-response electrical heaters based on ultraflexible, heat-resistant, and mechanically strong aramid nanofiber/ Ag nanowire nanocomposite papers. ACS Nano 13(7), 75787590 (2019). https://doi.org/10.1021/acsnano.9b00434

62. L. Zhang, H. Deng, Q. Fu, Recent progress on thermal conductive and electrical insulating polymer composites. Compos. Commun. 8, 74-82 (2018). https://doi.org/10.1016/j.coco. 2017.11.004 